\title{
PAGES Focus 3: A new Initiative on past Human Impacts
}

The main thrust of the PAGES program hitherto has been towards a better understanding of past climate change and its implications for the future. Focus 3 , which is concerned with the past impact of human activity on ecological and hydrological systems, has never been fully articulated, though significant progress has been made in one specific area - human impact on fluvial systems- thanks to the efforts of Bob Wasson. At the recent PAGES SSC meeting, held in Pallanza, N. Italy, June 19-21, an overall strategy was presented and endorsed. This strategy envisages seven interlinked themes as set out below. Their coordination and their articulation as PAGES Activities and Tasks will be the responsibility of a small steering group soon to be identified. The goals of the paleoscientists involved in this exercise will include the following:

- To define, promote and pursue areas of shared interest between PAGES and other IGBP project elements.

- To contribute fully to the PAGES, and eventually IGBP, syntheses currently being undertaken

- To push the development of these themes soundly forward toward and during the next stage of IGBP's work from the year 2000 onwards.

The themes briefly noted below have yet to be fully defined and, in some cases, leaders have yet to be identified. A work- shop to develop science and implementation plans will be held in late 98 / early 99 Meanwhile, colleagues interested in contributing to this aspect of PAGES work are invited to contact the IPO and its Executive Director (oldfield@pages.unibe.ch) who will be responsible for the overall coordination of Focus 3 in its early stages.

\section{Seven themes}

The themes envisaged at this stage are as follows:

\section{The history of greenhouse gas exchanges between the terrestrial biosphere and atmosphere}

These exchanges are of major concern in relation to understanding present day and future carbon dioxide and methane fluxes and budgets. There is an urgent need to improve our insight into the way past changes in land cover and hydrology have affected the fluxes and reservoirs over the non-marine part of the biosphere. The task spans a range of themes, from the past carbon balance in wetlands, to the impact of both deforestation and reforestation on $\mathrm{CO}_{2}$ fluxes at both regional and global scales. Moreover, there is a strong case for broadening it to include a concern with nitrogen species. One major advantage of undertaking this task for the past lies in the way in which it can be linked, on a range of timescales, to the trace gas record in ice cores. The task is seen as contributing to the GAIM led 'paleo-trace-gas challenge' and as helping to forge links between PAGES and other IGBP Project Elements, especially GCTE, BAHC and IGAC.

\section{Past biogeochemical fluxes within fluvial systems}

This theme is closely linked to the next two and it embraces the concerns of the BLOP report (Modelling the Transport and Transformation of Terrestrial Materials to Freshwater and Coastal Ecosystems) as well as one of the main tasks for PAGES within the IGBP inter project initiative on 'Continental Aquatic Systems'. It is also currently being actively promoted by the LUCIFS project (Land Use and Climate Impacts on Fluvial Systems during the Period of Agriculture) led by Bob Wasson. Further information on this project is contained in an article in this Newsletter on page 14 .

\section{The historical context of multiple threats to both aquatic and terrestrial ecosystems}

Climate variability and future climate change are only one factor, and perhaps often not the most important factor, in the complex of threats facing both terrestrial and aquatic ecosystems. Studies of soil erosion, surface

Open Science Meeting, continued from page 11

A wide-ranging discussion followed in which the issues raised included:

- lack of opportunities for developing and funding effective links between the areas of environmental and social sciences whose collaboration is essential to the emerging global change research agenda.

- the need, in an increasingly data-rich research environment, to focus on critical time intervals and processes.

- a request to IPCC for a clearer identification of the burning, unresolved questions to which paleo-research should make a crucial contribution. Suki Manabe, drawing up his own list, suggested the following:

- can we solve the problem of the 'missing carbon sink' through a concerted attack using the stable isotopes of carbon to better characterise the sinks, sources and fluxes?

- will global warming really reduce surface salinity in the North Atlantic?

- which are the key aerosols in climate feedback and what are their effects? - what are the most important observa- tional activities required to develop and validate models?

- the need for present day calibration to be linked to paleo-research projects. This may be one of the ways in which, given adequate coordination, essential monitoring programmes can be sustained.

- the challenge of incorporating hypothesis testing in the present research agenda, for example to test the possible role of marine biota in climate forcing.

- the urgent need, in some parts of the world, for more AMS radiocarbon measurement facilities worldwide, both for dating and for developing further the 'tracer' role of radiocarbon.

- the strong desirability of broadening the PAGES research agenda to encompass more fully other aspects of past global change than climate.

- the need to increase awareness of the relative magnitudes of changes in large scale averages. For example, the 'Little Ice Age' had major impacts, yet, over the northern hemisphere as a whole, appears to have been only 0.5 to $1^{\circ} \mathrm{C}$ cooler than the last few decades. In contrast, the IPCC worst case envisages a rapid warming of up to $4^{\circ} \mathrm{C}$.

The leading points made by the panel members and the ensuing discussion provided ample justification for regarding PAGES research as a vital contribution to understanding future environmental change and its human implications. They also provided many pointers to the ways in which the existing research agenda can be shaped in order to increase its impact and relevance. Our central task is to use the record from the past to shed light on what predicted future changes will actually mean for a still rapidly growing world population for which the goals of sustainable development and the realities of environmental change will be intimately linked.

\section{Frank Oldfield}

Executive Director, PAGES IPO

oldfield@pages.unibe.ch 
water acidification and eutrophication as well as the amelioration of the effects of these processes requires that they be set in historical context. Lake sediments serve as archives of all these processes, including the more recently recognized threats posed by increased nitrogen deposition and contamination by a range of 'new' compounds. Well-focused paleodata is essential for setting present day monitoring in time context as well as for model evaluation. This may be best illustrated by work on the history of acidification and eutrophication carried out world-wide over the last two to three decades in response to urgent environmental problems. This theme clearly interacts strongly with parts of the GCTE and LUCC science agendas.

\section{Historical perspectives on water quality/ lake ecosystems}

This theme is closely linked to the ones above and is, for the moment, separated from 3 only because it represents a significant part of PAGES input to the IGBP "Continental Aquatic Systems' inter-project already mentioned. It has now been accepted as a PAGES Activity within Focus 3, led by Rick Battarbee.

\section{Non-linear ecosystem changes in the geologically recent past}

Many researchers within the IGBP community rely on developing models of change through time by postulating temporal linkages between the different elements in a spatial mosaic. This approach has both strengths and weaknesses. One of the latter arises from the problem of rapid non-linear shifts in ecosystem function once key thresholds (e.g. in water retention or nutrient cycling) are transgressed. The paleo record is rich in evidence for such changes and there is a need to generate a better awareness of their causes and effects especially where the record from the past can be used to improve our understanding of highly stressed ecosystems at the present day.

\section{Global and regional time-slice reconstructions}

The well-developed BIOME 6000 program is an example of this type of activity. The need now is for reconstructions, especially of terrestrial vegetation and land cover, for the last 200 - 300 years, the period of rapidly accelerating change and human impact. This will call for an integration of paleorecords from sources as diverse as pollen analyses and past tax returns, and it will contribute directly to the needs of GCTE and LUCC, as well as to the theme identified under 1 above. It will be one of the themes in a joint PAGES-LUCC workshop planned for November 1998.

\section{The historical context of contemporary and future changes in areas of high 'value' and/or vulnerability}

In several cases, LUCC and GCTE, for example, envision a detailed focus on a specific region. In one case at least, the LBA in Amazonia, there is a coordinated IGBP activity across a wide range of Project Elements. Where such regions are defined, whether as transects across ecozones or as areas of concentrated research, PAGES can play an important role in placing changes in present day ecosystems and in the hydrology of the region in a longer time perspective. This can provide a dynamic baseline for future monitoring, an insight into processes operating on timescales longer than the span of available direct observations, and a quantitative estimate of the range of natural climate variability in the recent past. The capacity to do this will differ in different parts of the world, since it depends on the presence of suitable paleo-archives, ideally high resolution ones with records that come through continuously to the present day. Full exploitation of these rests on an acknowledgement that PAGES shares the task of understanding processes rather than simply providing 'historical reconstructions'. Many key processes in the climate system, at the ecosystem level and within hydrological systems, operate on decadal to century timescales. Establishing their functioning and their effects has an important role to play in understanding present and future earth system dynamics on all spatial scales.

\section{An eye on the future}

The themes outlined above must be a crucial part of the overall strategies of PAGES and the IGBP for several reasons. From the PAGES standpoint, we have to realize that no amount of paleoreconstruction can fully prepare us for future changes and future impacts operating on the present day landscape. The effects of human activity over the last ca. 200 years have led to transformations much more significant than those resulting from climate change. They have endowed us with a 'no-analogue' biosphere as the canvas upon which future climate changes and human activities will interact. Moreover, this no-analogue biosphere is the point of departure for the future part of the global experiment to which increasing greenhouse gas concentrations in the atmosphere will contribute. To consider this complex and rapidly changing canvas without regard to its antecedents is not a realistic enterprise. PAGES FOCUS 3 is therefore a research domain within which, as the messages from the past overlap the observations of the present, we may hope to develop and optimize the interactions between many aspects of IGBP's endeavors. Human activities are as much drivers of con- temporary and future environmental change as are anticipated changes in climate. The interplay between the two types of forcing is of vital concern and the history of their interaction is ignored at our peril, especially in the realm of future impacts and their integrated assessment. In this respect, the proposed FOCUS 3 initiatives are a response to human needs as well as to the emerging research agendas of national and international funding agencies.

\section{A call for participation}

The various themes outlined above are closely interwoven and will require a coordinated network approach for their realization. We can illustrate this simply by identifying the key archives for many of the themes, namely lacustrine and high resolution, near-shore marine sediment records of changing land cover, associated biogeochemical fluxes and their impacts on both terrestrial and aquatic ecosystem structure and function. There are other vital linking concerns. The concept of sustainability has no realistic meaning unless it accommodates what we know of past climate variability, human activities and the consequences of their interaction. Equally, development of the themes proposed is a prerequisite for model evaluation, which, in turn serves to improve predictive capability.

The first task is to identify a cohort of potential participants ready to share the challenges implicit in this proposal. An immense volume of highly relevant data already exists. One of our main roles must be to evaluate, filter, coordinate and systematize what is already known, as it bears on the key issues already partly identified in the outlines above. This 'prospectus' therefore comes with a request that all interested scientists contact us in the PAGES Office in Bern with an outline of their own perspectives and of the potential roles they may be prepared to play. In some cases, as for example in the case of aquatic ecosystems and of human impact on fluvial systems, the leaders (Rick Battarbee and Bob Wasson respectively) have been identified and work is already in progress. In other cases, there is a pressing need to identify those who will be prepared to play a crucial, active role in coordination and leadership. Again, we are putting this proposal forward in an effort to solicit both potential volunteers and nominees. By joining in such an effort, our hope is that you will not only contribute to making the best possible use of current knowledge and ongoing research; you will also be helping to define key aspects of the global change research agenda for the future.

\section{Frank Oldfield}

Executive Director, PAGES IPO

oldfield@pages.unibe.ch 